\title{
Age- and sex-related differences in temporal judgments to visual stimuli: Support for hemispheric equivalence
}

\author{
LENORA N. BROWN and ROBERT S. SAINSBURY \\ University of Calgary, Calgary, Alberta, Canada
}

\begin{abstract}
Light-emitting diodes in a visual half-field display were employed to examine hemispheric asymmetries in temporal resolution among young and older adults. Participants judged whether pairs of spatially separated diodes were illuminated simultaneously. No visual field threshold differences emerged for either age group, thus supporting hemispheric equivalence. Older adults had significantly higher thresholds than did younger adults, regardless of spatial location. The results further revealed that older females had significantly higher thresholds than did older males, younger males, and younger females. The results further revealed sex differences, favoring females, when interhemispheric transfer times (IHTTs) were examined for a central bilateral presentation. However, sex effects were not revealed when IHTTs were examined for a peripheral bilateral presentation, indicating a disadvantage for older females.
\end{abstract}

Efron (1963a) was among the first to report a lefthemisphere advantage for temporal processing of visual and tactile stimuli. Efron employed the Poffenberger (1912) paradigm and found that visual stimuli had to be presented to the left visual field 3-4 msec before right visual field presentation in order for the individual to perceive the stimulus events as simultaneous. Likewise, Efron found that left-sided tactile stimuli had to precede right-sided stimuli by a few milliseconds for the perception of simultaneity to occur. He reasoned that the onset asynchrony of stimuli (i.e., left before right presentation) was necessary for simultaneity judgments, since it required time for the information projected to the right hemisphere (left-hemispace stimulation) to transfer to the left hemisphere via the corpus callosum. The left hemisphere was posited as a serial processor, since it may have specialized mechanisms that may represent evolutionary precursors to lateralized speech perception and language (Fitch, Brown, O'Connor, \& Tallal, 1993). Given that the ability to understand language involves the sequencing of spoken words, whereas the production of coherent sentences involves the proper sequencing of words, the left hemisphere has been viewed as a serial processor (Efron, 1990).

This research was supported by the Research Services, University of Calgary. The authors extend their thanks to Michael Corballis, Gina Geffen, and Don Kline for their advice and recommendations. The authors especially thank Don Kline for sharing his expertise in regard to illumination levels and the aging visual system and thank Malcolm Bertram, Department of Geology and Geophysics, University of Calgary, for assisting in the construction of the research equipment so that the equipment operated with great precision. Correspondence should be addressed to L. N. Brown, Department of Psychology, University of Calgary, 2500 University Dr., Calgary, AB, T2N 1N4 Canada (e-mail: lnbrown@ucalgary.ca).
A left-hemisphere locus for temporal judgments was also reported in the auditory system. Several types of stimuli have been employed to examine temporal abilities in the auditory system. A left-hemisphere advantage has been reported for Morse code patterns (Papcun, Krashen, Terbeek, Remington, \& Harshman, 1974), auditory clicks (Mills \& Rollman, 1979), and dichotic tones (Divenyi \& Efron, 1979). In addition, Fitch et al. (1993) demonstrated a left-hemisphere advantage in rats for better discrimination of time sequences.

In the tactile modality, however, more recent findings tend to support a hemisphere equivalence model. Although earlier studies (e.g., Bakker \& Van der Kleij, 1978; Hammond, 1981; Nachshon \& Carmon, 1975) demonstrated a left-hemisphere advantage for the sequencing of tactile stimulation, more recent studies (Brown \& Sainsbury, 2000; Clark, Balfour, \& Geffen, 1989; Clark \& Geffen, 1990; Geffen, Mason, Butterworth, McLean, \& Clark, 1996; Geffen, Rosa, \& Luciano, 2000; Mason \& Geffen, 1996) have demonstrated no hemispheric lateralization for making temporal judgments when the hands are placed midline.

The findings of vision studies are less clear than the findings of tactile studies. Nicholls (1994) examined hemispheric asymmetries in the detection of simultaneously and successively presented stimuli (pairs of light-emitting diodes, LEDs) to either the left visual field (LVF) or the right visual field (RVF) (i.e., a bilateral condition was not included). Thus, stimuli presented in the LVF would be projected to the right hemisphere, and stimuli presented in the RVF would be projected to the left hemisphere. Nicholls reported a RVF advantage for judgments of simultaneity. Corballis (1996) investigated temporal judgments for successively and simultaneously presented visual stimuli in 
both bilateral (LVF and RVF presentations) and unilateral (LVF or RVF presentations) conditions. Although he reported a left-hemisphere advantage in the bilateral presentation, a left-hemispheric locus was not revealed when the stimuli were presented in either the left or the right hemifield (i.e., unilateral presentations). Corballis concluded that the lack of a significant difference between visual fields implies that no interhemispheric transfer occurred in the unilateral conditions. These findings suggest that both hemispheres are equally capable of making temporal judgments when stimuli are presented unilaterally. However, in contrast to Corballis (1996), Corballis, Boyd, Schulze, and Rutherford (1998) found a slight RVF advantage for males, but not for females. However, the data in the latter study did not reveal a left-hemisphere advantage when stimuli were presented bilaterally. Corballis et al. also reported that participants demonstrated better discrimination in unilateral presentations than in bilateral presentations.

Given the findings of these studies, it is doubtful that temporal judgments take place exclusively in the left hemisphere. The findings of temporal processing studies examining unilateral brain damaged individuals have not resolved the inconsistencies reported in the lateralization literature. Earlier studies, including Efron's, demonstrated deficits in temporal processing of patients with left temporal lobe lesions (Blumstein, Baker, \& Goodglass, 1977; Blumstein, Cooper, Zurif, \& Caramazza, 1977; Chedru, Bastard, \& Efron, 1978; Efron, 1963c; Lackner \& Teuber, 1973; Swisher \& Hirsh, 1972; Tallal \& Newcombe, 1978). Thus, originally, Efron (1963a, 1963b) proposed that the left hemisphere may be viewed as a serial processor, but he has since retracted this conclusion since he found that deficits in processing temporal stimuli were not restricted to left-hemisphere damage. Sherwin and Efron (1980) found significantly elevated thresholds in temporal ordering abilities for both left- and right-hemisphere damaged patients. They argued that deficits in temporal processing following damage to the right hemisphere were underestimated. In addition, Goldman, Lodge, Hammer, Semmes, and Mishkin (1968) found that patients with left temporal lobectomies were significantly impaired in detecting flickering light from continuous light (critical flicker frequency), and patients with right temporal lobectomies also demonstrated impairment but to a lesser degree. In contrast, Eals (1987) employed an apparent-motion task and found a right-hemisphere advantage for normal adults.

With respect to lateralization and aging, it has been proposed that information processing mediated by the right hemisphere, relative to the left hemisphere, declines with advanced age (Boll, 1974; Goldstein \& Shelly, 1981; R. C. Johnson et al., 1979; Klisz, 1978; Meudell \& Greenhalgh, 1987). That is, verbal abilities, which tend to be mediated by the left hemisphere, remain relatively stable across adulthood, whereas nonverbal abilities (e.g., visuospatial), which tend to be mediated by the right hemisphere, decline with advanced age (see Horn, 1982; Horn \& Hofer, 1992). The differential decline in verbal and nonverbal abilities initiated the hypothesis that the right hemisphere ages more rapidly than does the left hemisphere (Goldstein \& Shelly, 1981). However, the findings of cognitive and perceptual studies that have examined cerebral lateralization among the elderly are inconsistent. Several studies do not provide evidence for an age-related differential decline, favoring the left hemisphere (Borod \& Goodglass, 1980; Brown \& Sainsbury, 2000; Cherry, Hellige, \& McDowd, 1995; Hoyer \& Rybash, 1992; Nebes, Madden, $\&$ Berg, 1983), whereas other studies report an age-related differential decline, favoring the left hemisphere (Boll, 1974; Goldstein \& Shelly, 1981; R. C. Johnson et al., 1979; Klisz, 1978; Meudell \& Greenhalgh, 1987). However, other factors, such as task complexity, familiarity of task and materials, and speed (timed tasks), have been noted to affect age differences in performance (see Horn \& Hofer, 1992).

There is much evidence, however, that temporal processing abilities tend to decline with advanced age, especially with respect to the visual system. Several studies have reported an age-related decline in temporal resolution for visual stimuli (i.e., critical flicker frequency; Coppinger, 1955; Kline, Ikeda, \& Schieber, 1982; McFarland, Warren, \& Karris, 1958; Schieber \& Kline, 1982). However, it is difficult to determine the degree to which the agerelated decline in temporal visual processing is attributed to the functioning of the optical components or to neural functioning (for a review, see Brannan, 1992). However, there is evidence that neural functioning contributes to some of the age-related visual deficits in temporal resolution. That is, age-related deficits are not significantly reduced when young and older adults are equated for optical changes (C. A. Johnson, Adams, \& Lewis, 1989; Whitaker $\&$ Elliott, 1992), suggesting that the loss in temporal resolution with age may be strongly associated with neural decline.

The methods used to study adult age differences in temporal visual processing have yet to examine this area from a lateralized perspective. It is not known whether there is an age-related right-hemisphere decline in temporal processing of visual information. One of the challenges with lateralization studies is matching the stimuli that are projected to each hemisphere for complexity and familiarity. A lateralization study that employed the same testing material for both hemispheres would provide valuable information in regard to the right hemi-aging hypothesis. Few studies have included both unilateral and bilateral conditions (Brown \& Sainsbury, 2000; Clark \& Geffen, 1990; Corballis, 1996; Geffen et al., 1996) when investigating temporal processing asymmetries.

In the present study, we examined temporal processing abilities of young and older adults by employing a lateralized visual task (i.e., Poffenberger paradigm with no reaction time measures). We employed a visual half-field task to present visual stimuli that was similar (i.e., diameter of the six stimuli, spatial separation of stimuli, and viewing distance) to that used by Corballis (1996). This method minimized memory demands and eliminated task complexity, which has been related to age-related deficits in task performance. Visual simultaneity thresholds were 
examined to determine the temporal processing proficiency of each cerebral hemisphere for young and older adults. Thus, this paradigm reveals whether temporal processing deficits among the elderly are lateralized to one hemisphere (i.e., right hemi-aging hypothesis). Finally, by employing Poffenberger's paradigm, we estimated interhemispheric transfer times (IHTTs) by taking the difference in simultaneity thresholds between unilateral and bilateral conditions, in order to determine whether the transfer speed of visual information is affected by normal aging and/ or sex.

Briefly, the literature is inconsistent regarding whether there are age or sex differences in the size of the corpus callosum. Anatomical differences in the corpus callosum may have implications in the function of interhemispheric transfer (Hoptman, Davidson, Gudmundsson, Schreiber, $\&$ Ershler, 1996). Age-related structural reductions in the size of the corpus callosum have been identified (Allen, Richey, Chai, \& Gorski, 1991). As well, Witelson (1989) reported an age-related loss in callosal size in men, but not women. Salat, Ward, Kaye, and Janowsky (1997) found that, although older women had a larger posterior section of the corpus callosum than did men, only women showed age-related atrophy of the anterior and middle sections of the corpus callosum. Still other studies have reported no age-related reductions in callosal anatomy after adjustments for head size were made (S. C. Johnson, Farnworth, Pinkston, Bigler, \& Blatter, 1994; Rauch \& Jinkins, 1994). With respect to sex differences, several studies have reported a larger corpus callosum among females than among males (i.e., a larger splenial region of the corpus callosum; Davatzikos \& Resnick, 1998; Holloway \& de Lacoste, 1986). Since the splenium consists of fibers from the parietal and occipital regions for interhemispheric transfer (de Lacoste, Kirkpatrick, \& Ross, 1985), it is inferred that the greater number of fibers may result in better interhemispheric communication. However, more recently, Gur et al. (1999) found that men had a greater volume of white matter than did females, suggesting that the transfer of information within the brain would be faster for men. This paradigm will reveal whether there are sex differences in IHTTs and whether the overall age-related reduction in neural conduction applies to IHTT.

One goal of the present research was to determine whether both cerebral hemispheres are equally capable of processing fine visual temporal information and whether this pattern holds for the elderly. The second goal of the present study was to determine whether there is an agerelated differential decrement in temporal processing abilities for visual information. The third goal was to determine whether there are sex differences in visual temporal abilities and/or IHTTs.

\section{METHOD}

\section{Participants}

A total of 52 participants volunteered. The young group consisted of 13 males and 13 females and ranged in age from 20 to 40 years $(M=27.73$ years, $S D=7.08)$. The older group consisted of 13 males and 13 females from 60 to 80 years of age $(M=68.65$ years, $S D=6.12$ ). Each participant was tested in one session lasting 50-75 min. Participants gave informed consent.

All participants were required to be right-handed, have English as their native and primary language, have full use of both hands, and never to have been under a doctor's care for a neurological, psychiatric, or severe illness. The participants were questioned and excluded if they reported having any visual impairment (e.g., cataracts, glaucoma) and/or any substance abuse illnesses. A near binocular acuity assessment (Landolt $\mathrm{C}$ eyechart), with corrected vision, was administered in order to acquire a binocular visual acuity score. Binocular Snellen visual acuity scores for the young group $(M=20 / 20.46$, $S D=3.72)$ were lower than those for the older group $(M=20 / 32.07$, $S D=5.89$ ). All participants were classified as right-handed according to Annett's primary handedness questionnaire (Annett, 1970).

The young group had significantly more years of formal education $(M=14.65$ years, $S D=1.90)$ than did the older group $(M=$ 13.14 years, $S D=2.77)[F(1,50)=5.33, p<.05]$. When asked to rate their overall health status, all of the participants reported that they were relatively healthy. There were no significant group or sex differences in the self-reports of overall health or the level of physical activity.

The study consisted of a questionnaire to acquire general background information, a handedness questionnaire, a near binocular visual acuity assessment, and threshold measurements for five blocks of trials for the visual task. The general background questionnaire consisted of questions regarding overall health, medication use, physical exercise, and educational level. The handedness questionnaire (Annett, 1970) consisted of the six primary questions and was used to assess hand dominance. In order to be classified as right-handed, the participants were required to verbally respond "right" to each question and to use only the right hand when simulating the activities that were mentioned on the questionnaire.

\section{Apparatus}

The visual stimuli consisted of six red LEDs, $5.0 \mathrm{~mm}$ in diameter, which were placed on a horizontal plane against a white background $(81 \times 99 \mathrm{~cm})$. As can be seen in Figure 1, the LEDs were equally spaced at $33 \mathrm{~mm}$ (center to center) and symmetrically arranged with respect to the center of the visual display. A black cross in the center of the screen served as the central fixation point. Three LEDs were positioned on each side of the black cross, for a total of six paired locations. The LO (left outer) pair consisted of LEDs 1 and 2, the LI (left inner) pair consisted of LEDs 2 and 3, the Bil 1 (bilateral 1) pair consisted of LEDs 3 and 4, the RI (right inner) pair consisted of LEDs 4 and 5, the RO (right outer) pair consisted of LEDs 5 and 6, and the Bil 2 (bilateral 2) pair consist of LEDs 2 and 5.

The viewing distance was $57 \mathrm{~cm}$, so that each centimeter of the display subtended $1^{\circ}$ at the participant's eyes. Therefore, the pairs of LEDs were separated by $3.3^{\circ}$ of visual angle. For Bil 1, each LED (3 and 4) was placed $1.65^{\circ}$ from fixation. This amount of separation was sufficient, such that each LED was projected to the contralateral hemisphere (Fendrich \& Gazzaniga, 1989). For Bil 2, each LED ( 2 and 5) was placed $4.95^{\circ}$ from fixation. Thus, stimuli presented in the left hemifield were projected to the right hemisphere, and stimuli presented in the right hemifield were projected to the left hemisphere. A white side panel $(81 \times 51 \mathrm{~cm})$ was placed on each side of the white background visual display, in order to minimize any possible visual distractions.

The mean red light intensity emitted by the LEDs was $123 \mathrm{~cd} / \mathrm{m}^{2}$ (range $\left.=103-158 \mathrm{~cd} / \mathrm{m}^{2}\right)$ and was measured by a Minolta luminance meter prior to data collection. The luminance of the visual display, including the top, bottom, right-side, and left-side portions, was $46 \mathrm{~cd} / \mathrm{m}^{2}$. The luminances of all four quadrants of the display were relatively equal (i.e., range $=44-47 \mathrm{~cd} / \mathrm{m}^{2}$ ). Luminance was again measured approximately halfway through data collection. The mean light intensity emitted by the LEDs was $122 \mathrm{~cd} / \mathrm{m}^{2}$ (range = 

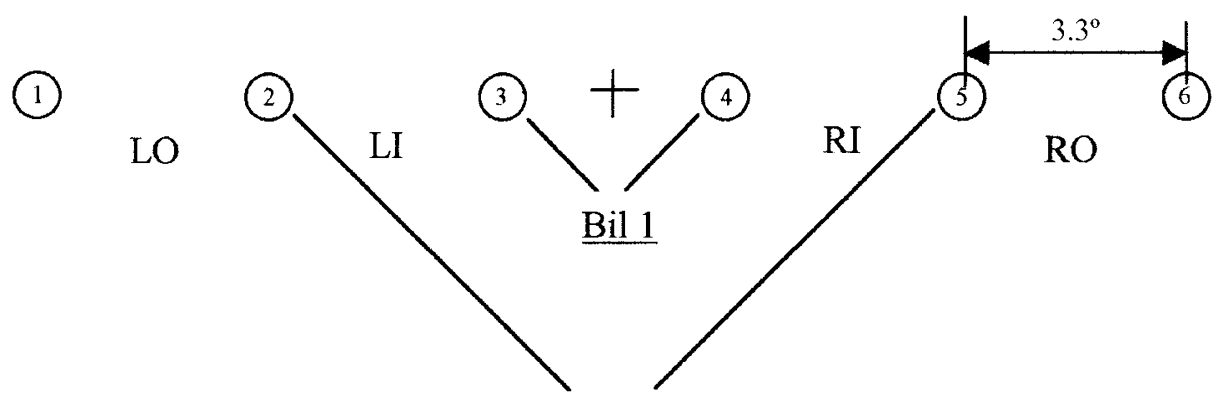

\section{$\underline{\text { Bil } 2}$}

Figure 1. Illustration of the six locations of LEDs and the six locations of the pairs of spatially separated LEDs. LO, left outer; LI, left inner; Bil 1, bilateral first pair; RI, right inner; RO, right outer; Bil 2, bilateral second pair.

$\left.105-159 \mathrm{~cd} / \mathrm{m}^{2}\right)$. The visual display luminance was $46 \mathrm{~cd} / \mathrm{m}^{2}$ (range $=44-48 \mathrm{~cd} / \mathrm{m}^{2}$ ).

The crystallized controlled oscillator in an external control box provided precise timing (highly stable $1-\mathrm{msec}$ output signal). The timing accuracy of the crystal control oscillator coupled with the 386 SX computer was within $5 \mathrm{msec}$. A Compaq 386 SX computer, coupled with the timing control, was used for stimulus delivery. The computer was programmed (Borland $\mathrm{C}++$, Version 2.0) with a modified version of the parameter estimation by sequential testing (PEST) algorithm, in order to control the delivery of the stimuli.

The PEST technique allows one to determine the value of thresholds in as few trials as possible (Findlay, 1978; Pentland, 1980). The testing values of the PEST technique are determined by a set of rules that operate by using a binary forced-choice (yes/no) paradigm. That is, the interval between the onset of the first stimulus to the onset of the second stimulus (stimulus onset asynchrony, SOA) was adjusted (increased or decreased) according to "yes" responses (perceived onsets of stimuli as simultaneous) and "no" responses (perceived onsets of stimuli as not simultaneous). The SOA step size changed by a constant value in association to "no" responses, and the SOA step size would increase by half of the previous SOA value in association to "yes" responses. Thus, a value change in SOA step sizes occurred only with step reversals associated with "yes" responses. The program terminated when the SOA step size reached $1 \mathrm{msec}$. For details regarding the PEST algorithm technique, see Brown and Sainsbury (2000).

A Sony SMF video camera (Model HVC 2800) was mounted at the rear of the display, facing the participant. The lens of the video camera was aligned with a small hole in the visual display that was just above the central fixation point. A monitor connected to the camera was used to observe the participant's eyes, in order to ensure that central fixation was maintained. A chinrest was used to fix the viewing distance at $57 \mathrm{~cm}$.

\section{Procedure}

The participants were instructed to respond "yes" if they judged the onset of the two LEDs as simultaneous and "no" if they judged the onset of the two LEDs as nonsimultaneous. Each participant completed six blocks of trials in one test session. Each block of trials consisted of six interwoven sequences of presentations that were randomized and controlled independently by the PEST program. Each session began with a practice block of trials, during which the smallest SOA value (the value at which a "no" response could confidently be established) was estimated. Fifteen milliseconds were added to this value, which served as the initial SOA value for the five test blocks. Therefore, the initial SOA value for the commencement of the test blocks was individualized for each participant. The initial
SOA step size was set at $10 \mathrm{msec}$, whereas the termination step size was set at $1 \mathrm{msec}$. The simultaneity threshold was the SOA value that terminated the PEST program.

In each block of trials, there were six paired locations for the illumination of the LEDs (i.e., LO, LI, Bil 1, RI, RO, and Bil 2). Illumination of the $\mathrm{LO}$ and LI pairs was projected to the right hemisphere, whereas illumination of the RI and RO pairs was projected to the left hemisphere. For the Bil 1 condition, illumination of LED 3 was projected to the right hemisphere, whereas illumination of LED 4 was projected to the left hemisphere. Finally, for the Bil 2 condition, illumination of LED 2 and LED 5 was projected to the contralateral hemisphere. For each SOA, the left LED was illuminated first for half of the trials, and the right LED was illuminated for the remaining half. The order in which the pairs of LEDs were illuminated and the direction of illumination (left before right, right before left) were randomly interwoven within each block of trials. Onsets differed, but offsets for each LED pair were simultaneous. The pair of LEDs remained illuminated for a duration of $1,000 \mathrm{msec}$ once both LEDs were illuminated.

The participant was instructed to focus on the fixation point (cross), with his/her chin resting on the chinrest. The participant was asked to respond "yes" if he/she judged the illumination onsets to be simultaneous and "no" if he/she judged the illumination onsets to be different. Verbal responses were recorded by the researcher. It could be argued that verbal responses may prime left-hemispheric processing. However, previous findings of several studies that have measured simultaneity thresholds by yes/no verbal responses have failed to demonstrate a left-hemisphere advantage (Brown \& Sainsbury, 2000; Clark \& Geffen, 1990; Geffen et al., 1996). It may be that verbal responses, or responses by hand movements, may have priming effects when reaction time is a dependent variable. The delivery of each stimulus pair was triggered following each recorded response. The participants were instructed that there were no right or wrong responses and to use their best judgment.

\section{RESULTS}

\section{Condition and Order Analyses}

For each participant, simultaneity thresholds were calculated from the mean of the five test blocks of trials for each of the six paired LED locations (LO, LI, Bil 1, RI, RO, and Bil 2). The mean simultaneity thresholds were subjected to a group (young, older) $\times$ sex $($ male, female $) \times$ condition (LO, LI, Bil 1, RI, RO, Bil 2) $\times$ order (left before right, right before left) mixed analysis of variance (ANOVA), 
Table 1

Mean Simultaneity Thresholds and Standard Deviations for the Young and Older Groups as a Function of Sex and Condition and Overall Mean Simultaneity Thresholds and Standard Deviations for Each Condition

\begin{tabular}{|c|c|c|c|c|c|c|c|c|c|c|c|c|}
\hline \multirow[b]{3}{*}{ Sex } & \multicolumn{12}{|c|}{ Condition } \\
\hline & \multicolumn{2}{|c|}{$\mathrm{LO}$} & \multicolumn{2}{|c|}{ LI } & \multicolumn{2}{|c|}{ Bil 1} & \multicolumn{2}{|c|}{ RI } & \multicolumn{2}{|c|}{ RO } & \multicolumn{2}{|c|}{ Bil 2} \\
\hline & $M$ & $S D$ & $M$ & $S D$ & $M$ & $S D$ & $M$ & $S D$ & $M$ & $S D$ & $M$ & $S D$ \\
\hline \multicolumn{13}{|c|}{ Young Group } \\
\hline Females & 32.79 & 11.92 & 25.58 & 10.01 & 26.10 & 9.54 & 24.95 & 8.90 & 30.14 & 10.78 & 33.00 & 14.27 \\
\hline Males & 28.57 & 9.26 & 24.58 & 9.87 & 29.24 & 9.12 & 22.72 & 7.39 & 27.73 & 8.62 & 34.59 & 14.42 \\
\hline \multicolumn{13}{|c|}{ Older Group } \\
\hline Females & 76.26 & 18.84 & 71.14 & 20.33 & 75.08 & 19.43 & 70.30 & 18.60 & 76.21 & 19.29 & 86.68 & 20.58 \\
\hline Males & 56.58 & 20.57 & 50.49 & 20.05 & 56.90 & 16.95 & 49.52 & 19.01 & 56.52 & 21.55 & 64.36 & 24.09 \\
\hline \multicolumn{13}{|c|}{ Overall } \\
\hline & 48.55 & 24.68 & 42.95 & 24.61 & 46.83 & 24.78 & 41.87 & 23.96 & 47.65 & 25.20 & 54.66 & 28.86 \\
\hline
\end{tabular}

with condition and order being repeated measures. Table 1 displays the mean thresholds for each condition by group and sex, as well as the overall mean thresholds for each condition.

A significant main effect for group $[F(1,48)=84.92$, $p<.001]$ was found. Simultaneity thresholds were significantly lower for young adults $(M=28.33 \mathrm{msec}, S D=$ $8.35)$ than for older adults $(M=65.84 \mathrm{msec}, S D=21.22)$. A significant main effect for $\operatorname{sex}[F(1,48)=6.70, p<.05]$ was obtained. Post hoc analysis of the significant sex effect revealed that the thresholds of males $(M=41.82 \mathrm{msec}$, $S D=20.42)$ were significantly lower than those of females $(M=52.35 \mathrm{msec}, S D=27.87)$.

A significant main effect for condition $[F(5,240)=$ $28.77, p<.001]$ was also obtained. For the condition main effect, comparisons of threshold values for paired locations were conducted (i.e., LO vs. LI; RO vs. RI; LO vs. RO; LI vs. RI; Bil 1 vs. Bil 2). Thresholds for the LO con- dition were significantly higher than those for the LI condition. Similarly, thresholds for the RO condition were significantly higher than those for the RI condition. Simultaneity thresholds were highest for the outer LED paired locations relative to those for the inner LED paired locations. No significant differences were found between thresholds for the $\mathrm{LO}$ and $\mathrm{RO}$ conditions and between thresholds for the LI and RI conditions.

The threshold values for the Bil 1 condition were significantly lower than those for the Bil 2 condition. Again, threshold values increased significantly from central locations to peripheral locations. There was no evidence of hemispheric asymmetry in simultaneity thresholds for any of the paired comparisons.

As can be seen in Figure 2, a significant group $\times$ sex interaction was obtained $[F(1,48)=5.66, p<.05]$. Further analysis of the interaction indicated that threshold values for both young males and young females were signif-

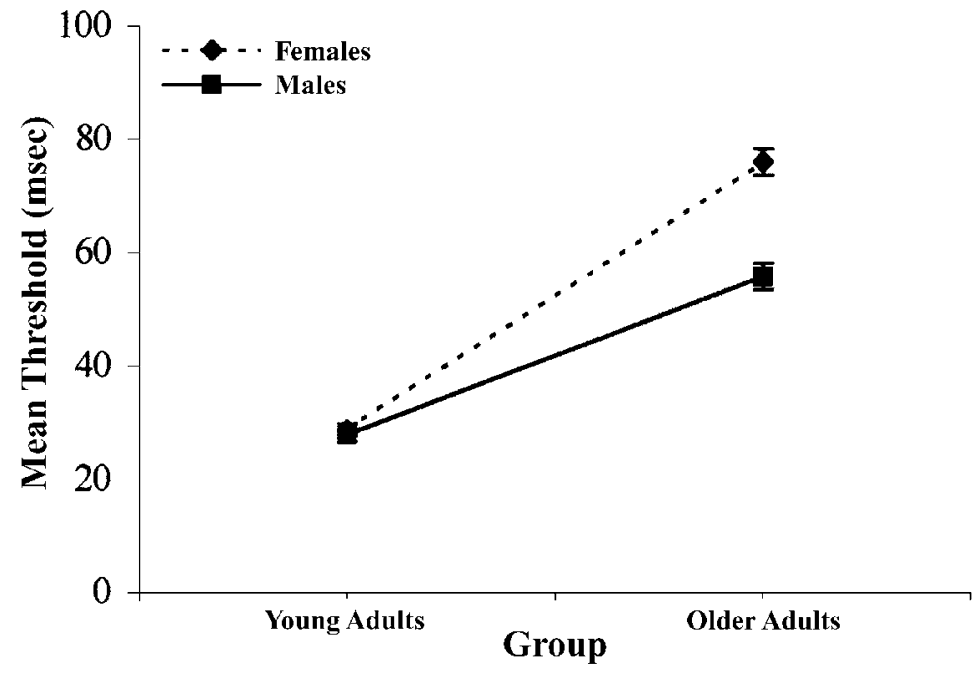

Figure 2. Mean simultaneity thresholds in the condition and order analysis for the young and older groups as a function of sex. 
icantly lower than those for older females and older males. It was also found that the threshold values for older males were significantly lower than those for older females. In an attempt to determine whether visual acuity measures may have influenced the sex effects in the older group, a correlational analysis of acuity scores was undertaken. The results indicated no relationship between simultaneity threshold values and visual acuity scores of older males and females $(Z=.2246, p>.05)$.

A significant group $\times$ condition $\times$ order interaction was also obtained $[F(5,240)=3.12, p<.01]$. Further analysis of the interaction, which is shown in Figure 3, revealed a stimulus order effect for the young adults under the LI and RI conditions only. Threshold values were lower when the onset of the left LED preceded the right LED for the LI location, whereas the reverse held for the RI location. That is, threshold values for the RI location were lower when the right LED preceded the left LED. This finding suggests that judgments of simultaneity are lower for the inner locations when the implied motion is directed toward central fixation. A left-hemisphere advantage would have been supported by lower thresholds when the illumination of the right LED preceded the left LED (i.e., leftward motion), relative to when the left LED preceded the right LED (i.e., rightward motion) in the bilateral conditions (Efron, 1963a). There was no evidence of hemispheric asymmetry in any of the statistical analyses.

\section{Bilateral Versus Unilateral Conditions}

In order to determine whether the bilateral simultaneity thresholds were significantly higher than the unilateral thresholds, which would indicate that integration of information involving bilateral presentations (interhemispheric transfer) is slower than that involving unilateral presentations (intrahemispheric transmission), two separate ANOVAs were conducted. The mean simultaneity thresh- olds of the Bil 1, LI, and RI were analyzed in the first analysis, and the mean simultaneity thresholds of the Bil 2, LO, and RO were analyzed in the second analysis. For the first analysis, mean simultaneity thresholds were subjected to a group (young, older) $\times$ sex (male, female $) \times$ condition (Bil 1, LI, RI) mixed ANOVA, with condition being a repeated measure. Refer to Table 1 for the threshold values.

A significant main effect for group $[F(1,48)=86.82$, $p<.001]$ was found. Simultaneity thresholds were significantly lower for young adults $(M=25.53 \mathrm{msec}, S D=$ $8.23)$ than for older adults $(M=62.24 \mathrm{msec}, S D=21.14)$. A significant main effect for $\operatorname{sex}[F(1,48)=6.38, p<.05]$ was also found. A simple analysis revealed that the thresholds of males ( $M=38.91 \mathrm{msec}, S D=19.55)$ were significantly lower than those of females $(M=48.86 \mathrm{msec}$, $S D=27.65)$. A significant effect for condition $[F(2,96)=$ $17.28, p<.001]$ was obtained. The analysis of the condition main effect revealed that the Bil 1 thresholds $(M=$ $46.83 \mathrm{msec}, S D=24.78$ ) were significantly higher than the LI thresholds $(M=42.95 \mathrm{msec}, S D=24.61)$ and the RI thresholds $(M=41.87 \mathrm{msec}, S D=23.96)$, which did not differ.

A significant group $\times$ sex interaction was obtained $[F(1,48)=6.34, p<.05]$. Further analysis of the interaction revealed that threshold values for both young males and young females were significantly lower than those for older males and older females. In addition, thresholds for the older females were significantly higher than those for the older males.

For the second analysis, mean simultaneity thresholds were subjected to a group (young, older) $\times$ sex (male, female) $\times$ condition (Bil 2, LO, RO) mixed ANOVA, with condition being a repeated measure. A significant main effect for group $[F(1,48)=78.68, p<.001]$ was found. Simultaneity thresholds were significantly lower for young

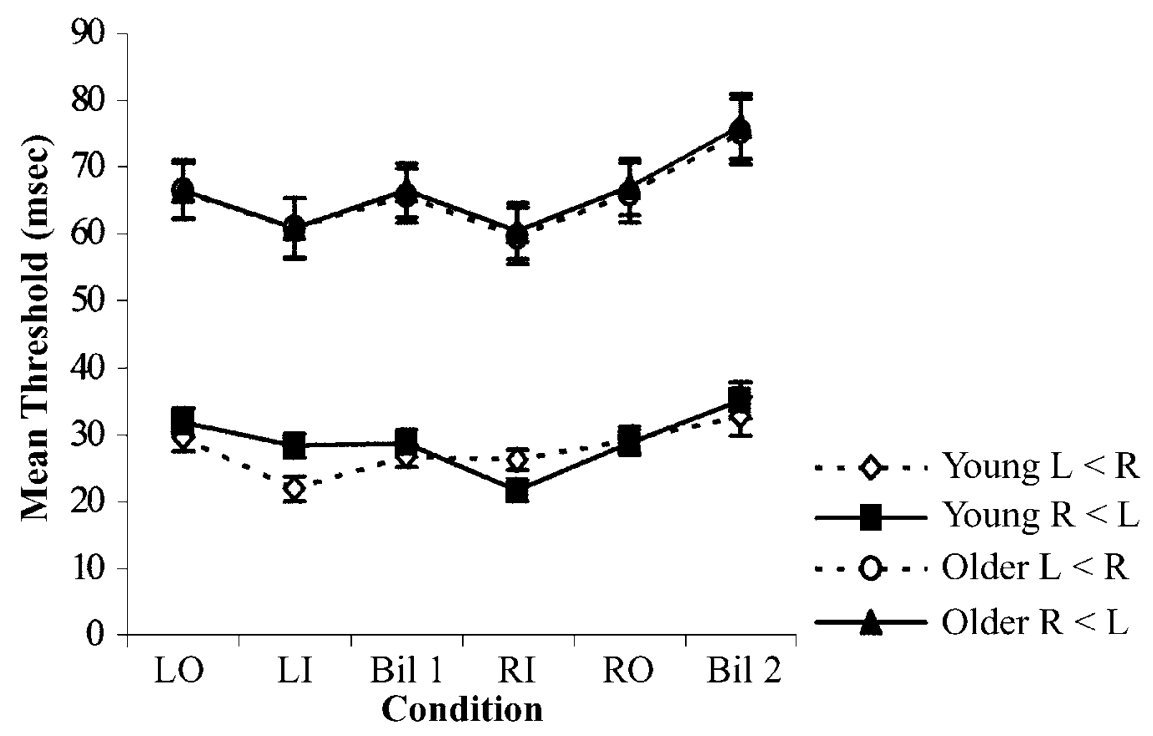

Figure 3. Mean visual simultaneity thresholds for the young and older groups as a function of condition and order. 
adults $(M=31.14 \mathrm{msec}, S D=10.99)$ than for older adults $(M=69.44 \mathrm{msec}, S D=23.05)$. A significant main effect for sex $[F(1,48)=6.63, p<.05]$ was obtained. A simple analysis revealed that the thresholds for males $(M=$ $44.73 \mathrm{msec}, S D=22.49)$ were significantly lower than those for females $(M=55.85 \mathrm{msec}, S D=28.76)$.

A significant main effect for condition $[F(2,96)=17.28$, $p<.001]$ was also obtained. The simple analysis revealed that the Bil 2 thresholds $(M=54.66 \mathrm{msec}, S D=28.86)$ were significantly higher than the LO thresholds $(M=$ $48.55 \mathrm{msec}, S D=24.68)$ and the RO thresholds $(M=$ $47.65 \mathrm{msec}, S D=25.20$ ), which did not differ.

As revealed in the previous ANOVA, a significant group $\times$ sex interaction was obtained $[F(1,48)=4.78$, $p<.05]$. Further analysis of the interaction revealed that threshold values for both young males and young females were significantly lower than those for older males and females. Thresholds for the older females were significantly higher than those for the older males. In order to determine whether the visual acuity scores between older males and older females were significantly different, a separate ANOVA for the older group was undertaken. Acuity scores for older males $(M=29.54, S D=5.17)$ were significantly lower than those for females $(M=34.62, S D=$ 5.62) $[F(1,24)=5.74, p<.05]$.

\section{IHTT Analyses}

Two estimates of the IHTTs for temporal visual processing were calculated by taking the difference of the means between the bilateral conditions and the unilateral conditions (i.e., Bil 1 and Bil 2 conditions). For the Bil 1 condition, the difference between the mean of the Bil 1 condition and the combined means of the LI and RI unilateral conditions were calculated. These estimates were subjected to a group (young, older) $\times$ sex (male, female) ANOVA. A significant main effect for sex was obtained $[F(1,48)=4.87, p<.05]$. Males $(M=6.24 \mathrm{msec}, S D=$ $5.44)$ had significantly longer IHTT estimates than did females $(M=2.60 \mathrm{msec}, S D=6.50)$. For the Bil 2 condition, the difference between the mean of the Bil 2 condition and the combined means of the LO and RO unilateral conditions were calculated. These estimates were subjected to a group (young, older) $\times$ sex (male, female) ANOVA. No significant effects were revealed. However, as can be seen in Figure 4, the IHTT estimates for the older females doubled in the Bil 2 condition relative to the Bil 1 condition, diminishing the significant sex effect that was revealed in the Bil 1 condition analyses. As well, the standard deviations nearly doubled from the Bil 1 condition to the Bil 2 condition for all four groups (Figure 4).

\section{Number of Trials Analysis}

Given the nature of the PEST technique, the determination of threshold values differs for each participant, thus producing a different number of trials for each participant. In order to examine whether the higher thresholds of the older group were associated with a greater number of trials, which may indicate unstable responses (e.g., responding "yes" to a 60-msec SOA separation, then "no" to a 65msec SOA value, and then responding "no" to a 60-msec SOA value), the mean number of trials across the five test blocks was subjected to a group (young, older) $\times$ sex (male, female) ANOVA. A significant effect for group was found $[F(1,48)=4.66, p<.05]$. Interestingly, the mean number of trials for the older group $(M=91.03$, $S D=16.43$ ) was significantly lower than the mean num-

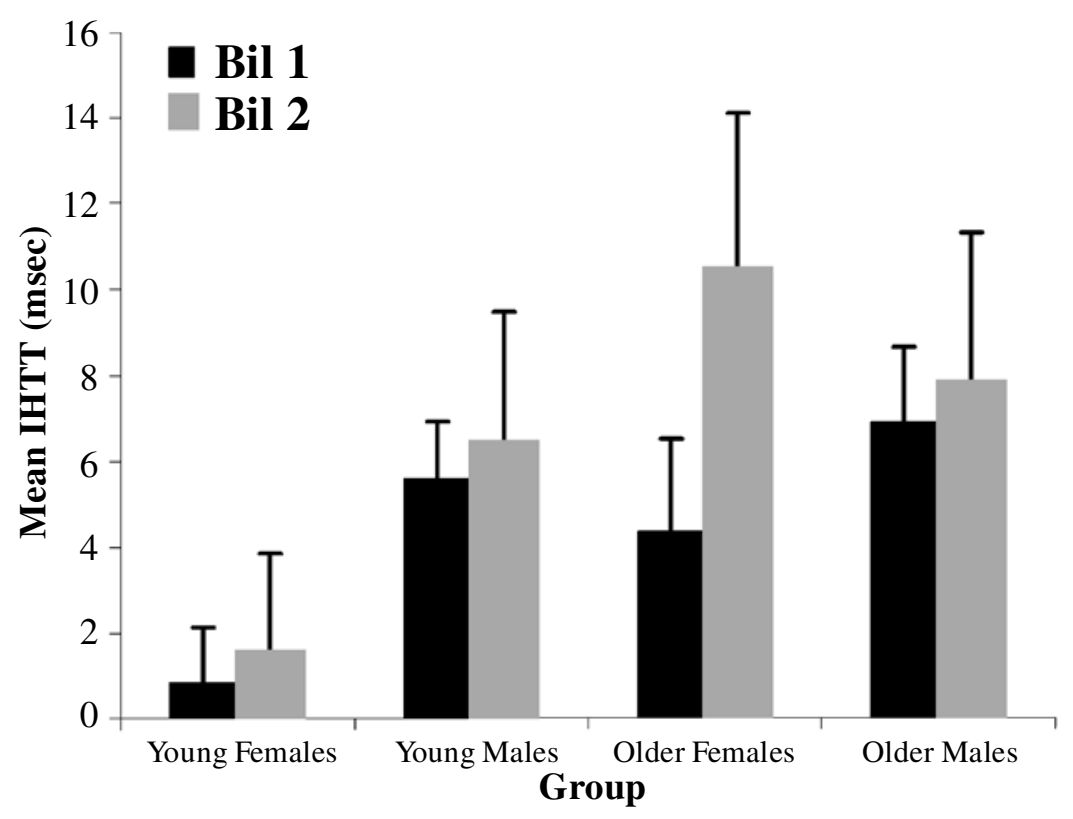

Figure 4. Mean IHTTs for the Bil 1 and Bil 2 conditions as a function of group and sex 
ber of trials for the younger group $(M=95.01, S D=$ 14.71). A significanteffect for sex also emerged $[F(1,48)=$ $9.80, p<.01]$. Males $(M=89.24, S D=13.30)$ had a significantly lower mean number of trials than did females $(M=100.78, S D=13.98)$. The results show that young females required significantly more trials than did all other groups in order to establish a simultaneity threshold.

\section{Correlational Analyses}

Correlations were conducted in order to determine whether age, educational level, medication use, and visual acuity were related to visual simultaneity thresholds. Four correlation matrices, one for each of the four groups (young females, young males, older females, older males), that included age, educational level, medication use, visual acuity measures, and visual simultaneity thresholds were examined. The absence of significant correlations between the variables and simultaneity thresholds indicates that simultaneity judgments were not related to visual acuity, medication use, and educational level.

\section{DISCUSSION}

The findings support the hemispheric equivalence model as proposed by Geffen et al. (1996). This is especially evident in that there were no significant threshold differences in the stimulus order in either bilateral condition (i.e., Bil 1, Bil 2). Comparisons of simultaneity thresholds for paired locations (i.e., LO vs. LI; RO vs. RI; $\mathrm{LO}$ vs. RO; LI vs. RI; Bil 1 vs. Bil 2) did not reveal a hemispheric advantage. In support of Corballis et al.'s (1998) results, the bilateral simultaneity thresholds were significantly higher than the unilateral simultaneity thresholds, suggesting that the integration of information involving interhemispheric transfer is slower than the integration of information involving intrahemispheric transmission.

The present results are not consistent with the earlier findings reported by Corballis (1996) and Nicholls (1994). Corballis reported a left-hemisphere advantage when stimuli were presented bilaterally, whereas Nicholls reported a right visual field (left-hemisphere) advantage for judgments of simultaneity when stimuli were presented unilaterally. However, more recently, Corballis et al. (1998) reported a left-hemisphere advantage for temporal judgments in males when stimuli were presented in the right visual field only. Their bilateral presentations failed to reveal a left- or right-hemisphere advantage. The present data are not inconsistent with those of Corballis et al. in that a left-hemisphere advantage was not exhibited in bilateral presentations.

The present findings indicate not only that both cerebral hemispheres are equally capable of processing visual temporal information but that the hemispheric equivalence for making judgments of simultaneity remains stable through adulthood.Evidence supporting hemispheric equivalence with advanced age was revealed by similar threshold values between the two unilateral conditions and by the similar threshold values for the order of stimulation in the bilateral presentations. Although the findings of the present study provide evidence for the uniform aging of the cerebral hemispheres (Borod \& Goodglass, 1980; Hoyer \& Rybash, 1992), the absence of an age-related differential hemispheric decline in judgments of simultaneity is some distance from the complex aging process of the cerebral hemispheres. Nonetheless, the present results indicate that this type of ability does not decline differentially between hemispheres. These results are consistent with the findings reported by Brown and Sainsbury (2000), in that there is an age-related decline in simultaneity judgments and that both cerebral hemispheres are equally capable of making temporal decisions regardless of age.

The present findings suggest that the right hemi-aging hypothesis may be artifact of methodology. That is, when the testing material employed for lateralization studies is not equated for complexity and familiarity, lateralization effects may emerge. Lifelong experience with verbal material (usually processed by the left hemisphere) may explain why left-hemisphere advantages are sometimes observed among the elderly.

The findings support the view that there is an age-related decline in the ability to make visual temporal judgments (Schieber \& Kline, 1982), regardless of location within the visual field. Visual simultaneity thresholds for both young and older adults appear to be consistent with values reported by other researchers. In the present study, an overall visual mean simultaneity threshold of $28 \mathrm{msec}$ was observed for the young adults and $66 \mathrm{msec}$ for the older adults. Corballis et al. (1998) reported that young adults began to discriminate nonsimultaneity consistently when the stimuli were separated by SOAs of $33 \mathrm{msec}$ or greater. In addition, Schieber and Kline reported above-chance performance (i.e., ceiling effects) at $45 \mathrm{msec}$ for young adults and $90 \mathrm{msec}$ for older adults in the discrimination of nonsimultaneity. The present results indicate that the age-related increase in simultaneity thresholds may be attributed to changes in the nervous system, relative to the age-related changes that typically occur in the properties of the eye itself (Elliott, Whitaker, \& MacVeigh, 1990; Kline et al., 1982). The illumination levels of the LEDs were sufficient such that visual abilities or acuity were not being tested. As well, visual acuity scores did not correlate with simultaneity thresholds.

Within in each condition, the present findings suggest that there is a significant age-related decrease in the ability to make simultaneity judgments in the visual modality. The results also revealed significantly poorer visual simultaneity thresholds for the older females, suggesting that females may be more susceptible to the age-related decline in the ability to time visual information than are males. The number of trials taken to establish simultaneity thresholds could not explain the sex or age differences in simultaneity thresholds. One would expect older females to have the highest number of trials, since they may be unsure of their responses. In fact, it was the youngerfemales who had the highest number of trials in order to establish thresholds. With respect to the sex effect in the older group, a correlational analysis indicated no relationship between simultaneity thresholds and visual acuity measurements for 
older males and females. However, the sex difference in visual acuity scores, favoring males, may be indicative of an early sign of age-related disease (e.g., cataract, macular degeneration) in females, which may have contributed to the sex difference in simultaneity thresholds. A difference in chronological age of older participants was also considered, such that the females may be older than the males. However, the ages for males and females in the older group appeared to be matched. Eight females were 61-67 years of age, 7 males were 61-69 years of age, 5 females were 70-80 years of age, and 6 males were 70-79 years of age.

The sex effects do support the findings reported by Atchley and Anderson (1998), in that a decline in visual motion detection performance, especially at the fovea area, was revealed for older females relative to older males. The authors proposed that their age and sex interaction for motion sensitivity may be explained by a greater loss of ganglion cells in central vision (M-type cells) for older females than for older males. However, in the present study, simultaneity thresholds of all participants increased from central to peripheral processing. It is interesting to note, however, that older females are not as proficient as older males in temporal processing or in the detection of motion for visual stimuli.

The IHTT estimates of $3.21 \mathrm{msec}$ for the younger adults in the Bil 1 condition and $3.99 \mathrm{msec}$ in the Bil 2 condition appear to be comparable to the IHTT estimates of 3$6 \mathrm{msec}$, which were reported by Poffenberger (1912), Efron (1963c), and Marzi, Bisiacchi, and Nicoletti (1991). The finding that IHTTs for females were significantly shorter than those for males in the Bil 1 condition supports the sex difference in callosal structure and size that was reported by Davatzikos and Resnick (1998) and Holloway and de Lacoste (1986). Although these results support the findings of faster IHTTs for females than for males (e.g., Potter \& Graves, 1988), they contradict the findings reported by Geffen et al. (2000), in that males were found to have faster IHTT estimates than females when tactile stimuli were examined.

Unfortunately, the consistency of the IHTT estimates for the older adults cannot be evaluated since other studies have failed to report such data. However, the data shown in Figure 4 suggest that older females are disadvantaged in IHTT when examining peripheral visual temporal processing (i.e., Bil 2 condition), relative to central visual temporal processing (i.e., Bil 1 condition). However, given the larger spatial separation for the Bil 2 condition relative to the Bil 1 condition, it may be that some proportion of the higher IHTT estimates may be due to the greater retinal travel distance for the Bil 2 condition. It is also possible that the higher Bil 2 IHTT estimates may be partially explained by attentional problems that are associated with widely spaced stimuli. Indeed, the interhemispheric transfer is an area that requires further research in order to gain a better understanding of why such mixed findings appear in the literature.

This study examined (1) age and sex differences in visual temporal processing from a lateralized perspective by using the same testing stimuli for both hemispheres so that task-related demands were equated (low complexity) and (2) age and sex differences in IHTTs. The results of this study indicate that both hemispheres are equally capable of processing visual temporal information and that hemispheric equivalence for judgments of simultaneity remains stable through adulthood. An age-related decline in the ability to make temporal judgments to visual stimuli was also revealed. When sex was examined, it was found that older females had the highest simultaneity thresholds, regardless of location. Finally, the results revealed that females had shorter IHTTs than did males when a central bilateral condition was examined. However, sex effects were not revealed when IHTTs were examined for a peripheral bilateral condition. The findings indicated that IHTT for older females is significantly longer for peripheral presentations relative to central presentations.

\section{REFERENCES}

Allen, L. S., Richey, M. F., Chai, Y. M., \& Gorski, R. A. (1991). Sex differences in the corpus callosum of the living human being. Journal of Neuroscience, 11, 933-942.

ANNETT, M. (1970). A classification of hand preference by association analysis. British Journal of Psychology, 61, 303-321.

Atchley, P., \& Anderson, G. J. (1998). The effect of age, retinal eccentricity, and speed on the detection of optic flow components. Psychology \& Aging, 13, 1-12.

BAKKeR, D. J., \& VAN DER KLeIJ, P. C. M. (1978). Development of lateral asymmetry in the perception of sequentially touched fingers. Acta Psychologica, 42, 357-365.

Blumstein, S. E., Baker, E., \& Goodglass, H. (1977). Phonological factors in auditory comprehension in aphasia. Neuropsychologia, 15, 19-30.

Blumstein, S. E., Cooper, W. E., Zurif, E. B., \& Caramazza, A. (1977). The perception and production of voice-onset time in aphasia. Neuropsychologia, 15, 371-383.

BoLL, T. J. (1974). Right and left cerebral hemisphere damage and tactile perception: Performance of the ipsilateral and contralateral sides of the body. Neuropsychologia, 12, 235-238.

Borod, J. C., \& Goodglass, H. (1980). Lateralization of linguistic and melodic processing with age. Neuropsychologia, 81, 79-83.

BRANNAN, J. R. (1992). Changes in temporal visual processing in normal aging. In J. R. Brannan, G. E. Stelmach, \& P. A. Vroon (Eds.), Advances in psychology: Applications of parallel processing in vision (pp. 119-134). Amsterdam: North-Holland.

Brown, L. N., \& SAInsbury, R. S. (2000). Hemispheric equivalence and age-related differences in judgments of simultaneity to somatosensory stimuli. Journal of Clinical \& Experimental Neuropsychology, 22, 587-598.

Chedru, F., Bastard, V., \& Efron, R. (1978). Auditory micropattern discrimination in brain damaged subjects. Neuropsychologia, 16, 141149.

Cherry, B. J., Hellige, J. B., \& McDowd, J. M. (1995). Age differences and similarities in patterns of cerebral hemispheric asymmetry. Psychology \& Aging, 10, 191-203.

Clark, C. R., Balfour, C., \& GefFen, G. M. (1989). Hemispheric processing of temporal information. International Journal of Comparative Psychology, 3, 73-95.

Clark, C. R., \& Geffen, G. M. (1990). Hemispheric equivalence for simultaneity judgments of somatosensory stimuli. Cognitive Neuropsychology, 7, 311-327.

Coppinger, N. W. (1955). The relationship between critical flicker frequency and chronological age for varying levels of stimulus brightness. Journal of Gerontology, 10, 48-52.

Corballis, M. C. (1996). Hemispheric interactions in temporal judgments about spatially separated stimuli. Neuropsychology, 10, 42-50.

Corballis, M. C., Boyd, L., Schulze, A., \& Rutherford, B. J. 
(1998). Role of commissures in interhemispheric temporal judgments. Neuropsychology, 12, 519-525.

Davatzikos, C., \& Resnick, S. M. (1998). Sex differences in anatomic measures of interhemispheric connectivity: Correlations with cognition in women but not men. Cerebral Cortex, 8, 635-640.

de Lacoste, M. C., Kirkpatrick, J. B., \& Ross, E. D. (1985). Topography of the human corpus callosum. Journal of Neuropathology \& Experimental Neurology, 44, 578-591.

Divenyi, P. L., \& EFron, R. (1979). Spatial versus temporal features in dichotic listening. Brain \& Language, 7, 375-386.

EALS, M. (1987). Asymmetric processing in perception of apparent movement. Neuropsychologia, 25, 429-434.

EFron, R. (1963a). The effect of handedness on the perception of simultaneity and temporal order. Brain, 86, 261-284.

EFrON, R. (1963b). The effect of stimulus intensity on the perception of simultaneity in right- and left-handed subjects. Brain, 86, 285-294.

EFron, R. (1963c). Temporal perception, aphasia and déjà vu. Brain, 86, 403-424.

EFrON, R. (1990). The decline and fall of hemispheric specialization. Hillsdale, NJ: Erlbaum.

Elliott, D., Whitaker, D., \& MacVeigh, D. (1990). Neural contribution to spatiotemporal contrast sensitivity decline in healthy ageing eyes. Vision Research, 30, 541-547.

Fendrich, R., \& Gazzaniga, M. S. (1989). Evidence of foveal splitting in a commissurotomy patient. Neuropsychologia, 27, 273-281.

FindLAY, J. M. (1978). Estimates on probability functions: A more virulent PEST. Perception \& Psychophysics, 23, 181-185.

Fitch, R. H., Brown, C. P., O’ Connor, K., \& Tallal, P. (1993). Functional lateralization for auditory temporal processing in male and female rats. Behavioral Neuroscience, 107, 844-850.

Geffen, G. [M.], Mason, C., Butterworth, P., McLean, S., \& Clark, C. R. (1996). Tactile simultaneity thresholds within and between hemispheres: The effects of hemispace. Cognitive Neuropsychology, 13, 257-276.

Geffen, G. [M.], Rosa, V., \& Luciano, M. (2000). Effects of preferred hand and sex on the perception of tactile simultaneity. Journal of Clinical \& Experimental Neuropsychology, 22, 219-231.

Goldman, P. S., Lodge, A., Hammer, L. R., Semmes, J., \& Mishinin, M. (1968). Critical flicker frequency after unilateral temporal lobectomy in man. Neuropsychologia, 6, 355-366.

Goldstein, G., \& Shelly, C. (1981). Does the right hemisphere age more rapidly than the left? Journal of Clinical Neuropsychology, $\mathbf{3}$, 65-78.

Gur, R. C., Turetsky, B. I., Matsui, M., Yan, M., Bilker, W., Hughett, P., \& Gur, R. E. (1999). Sex differences in gray and white matter in healthy young adults: Correlations with cognitive performance. Journal of Neuroscience, 19, 4065-4072.

Hammond, G. R. (1981). Finer temporal acuity for stimuli applied to the preferred hand. Neuropsychologia, 19, 325-329.

Holloway, R. L., \& De Lacoste, M. C. (1986). Sexual dimorphism in the human corpus callosum: An extension and replication study. Human Neurobiology, 5, 87-91.

Hoptman, M. J., Davidson, R. J., Gudmundsson, A., Schreiber, R. T., \& ERSHLER, W. B. (1996). Age differences in visual evoked potential estimates of interhemispheric transfer. Neuropsychology, 10, 163-271.

HorN, J. L. (1982). The aging human abilities. In B. B. Wolman (Ed.), Handbook of developmental psychology (pp. 847-870). Englewood Cliffs, NJ: Prentice-Hall.

Horn, J. L., \& Hofer, S. M. (1992). Major abilities and development in the adult period. In R. J. Sternberg \& C. A. Berg (Eds.), Intellectual development (pp. 44-99). Cambridge: Cambridge University Press.

Hoyer, W. J., \& RYBASH, J. M. (1992). Age and visual field differences in computing visual-spatial relations. Psychology \& Aging, 7, 339-342.

Johnson, C. A., Adams, A. J., \& Lewis, R. A. (1989). Evidence for a neural basis of age-related visual field loss in normal observers. Investigative Ophthalmology Visual Science, 30, 2056-2064.

Johnson, R. C., Cole, R. E., Bowers, J. K., Foiles, S. V., Nikaido, A. M., Patrick, J. W., \& Woliver, R. E. (1979). Hemispheric efficiency in middle and later adulthood. Cortex, 15, 109-119.

Johnson, S. C., Farnworth, T., Pinkston, J. B., Bigler, E. D., \&
Blatter, D. B. (1994). Corpus callosum surface area across human adult life span: Effect of age and gender. Brain Research Bulletin, 35, 373-377.

Kline, D. W., Ikeda, D. M., \& Schieber, F. J. (1982). Age and temporal resolution in color vision: When do red and green make yellow? Journal of Gerontology, 37, 705-709.

KLISZ, D. (1978). Neuropsychological evaluation of older persons. In M. Storandt, I. C. Siegler, \& M. F. Elias (Eds.), The clinical psychology of aging. New York: Plenum.

Lackner, J. R. \& Teuber, H. L. (1973). Alterations in auditory fusion thresholds after cerebral injury in man. Neuropsychologia, 11, 409415.

Marzi, C. A., Bisiacchi, P., \& Nicoletti, R. (1991). Is interhemispheric transfer of visuomotor information asymmetric? Evidence from a meta-analysis. Neuropsychologia, 29, 1163-1177.

Mason, C., \& Geffen, G. [M.] (1996). Temporal integration of events within and between hemisphere: The effects of hemispace. Cognitive Neuropsychology, 32, 97-108.

McFarland, R. A., Warren, A. B., \& Karris, C. (1958). Alterations in critical flicker frequency as a function of age and light:dark ratio. Journal of Experimental Psychology, 56, 529-538.

Meudell,P. R., \& Greenhalgh, M. (1987). Age-related differences in left and right hand skill and in visuo-spatial performance: Their possible relationships to the hypothesis that the right hemisphere ages more rapidly than the left. Cortex, 12, 431-445.

Mills, L., \& Rollman, G. B. (1979). Left hemisphere selectivity for processing duration in normal subjects. Brain \& Language, 7, 320-335.

NACHSHON, L., \& CARMon, A. (1975). Hand preference in sequential and spatial discrimination tasks. Cortex, 11, 123-131.

Nebes, R. D., Madden, D. J., \& Berg, W. D. (1983). The effect of age on hemispheric asymmetry in visual and auditory identification. $E x$ perimental Aging Research, 9, 87-91.

Nicholls, M. E. R. (1994). Hemispheric asymmetries for temporal resolution: A signal detection analysis of threshold and bias. Quarterly Journal of Experimental Psychology, 47A, 291-310.

Papcun, G., Krashen, S., Terbeek, D., Remington, R, \& Harshman, R. (1974). Is the left hemisphere specialized for speech, language and/or something else? Journal of the Acoustical Society of America, 55, 319-327.

Pentland, A. (1980). Maximum likelihood estimation: The best PEST. Perception \& Psychophysics, 28, 377-379.

Poffenberger, A. T. (1912). Reaction time to retinal stimulation with special reference to the time lost in conduction through nervous centers. Archives of Psychology, 23, 1-73.

Potter, S. M., \& Graves, R. E. (1988). Is interhemispheric transfer related to handedness and sex? Neuropsychologia, 26, 319-325.

RAUCH, R. A., \& JinKINS, J. R. (1994). Analysis of cross-sectional area measurements of the corpus callosum adjusted for brain size in male and female subjects from childhood to adulthood. Behavioural Brain Research, 64, 65-78.

Salat, D., Ward, A., Kaye, J. A., \& Janowsky, J. S. (1997). Sex differences in the corpus callosum with aging. Neurobiology of Aging, 18, 191-197.

Schieber, F., \& Kline, D. W. (1982). Age and the discrimination of visual successiveness. Experimental Aging Research, 8, 159-161.

SHERWIN, I., \& EFron, R. (1980). Temporal ordering deficits following anterior temporal lobectomy. Brain \& Language, 11, 195-203.

SWISHER, L., \& HirSH, I. J. (1972). Brain damage and the ordering of two temporally successive stimuli. Neuropsychologia, 10, 137-152.

TAllal, P., \& Newcombe, F. (1978). Impairment of auditory perception and language comprehension in dysphasia. Brain \& Language, 5, 1324.

WhitaKer, D., \& Elliott, D. B. (1992). Simulating age-related optical changes in the human eye. Documenta Ophthalmologica, 82, 307-316.

Witelson, S. F. (1989). Hand and sex differences in the isthmus and genu of the human corpus callosum: A postmortem morphological study. Brain, 112, 799-835.

(Manuscript received December 15, 2000; revision accepted for publication October 23, 2001.) 